\title{
Estudiantes universitarios y juego patológico. Un estudio empírico en la Universidad de Murcia
}

\section{University students and pathological gambling. A empirical research in the University of Murcia}

\author{
Ma Ángeles Jiménez Tallón, Carmen García Montalvo, \\ Marian Montero Jiménez y $\mathrm{M}^{\mathrm{a}}$ del Carmen Perea Pérez \\ Departamento de Personalidad, Evaluación y Tratamiento Psicológicos \\ Universidad de Murcia, España
}

Disponible online 31 de diciembre de 2011

\begin{abstract}
El objetivo de este trabajo ha sido investigar la presencia de características de ludopatía en una muestra de estudiantes universitarios. Para ello se estudió una población de 908 sujetos matriculados en diferentes facultades universitarias de la Universidad de Murcia y se les aplicó el Cuestionario de juego de South Oaks (South Oaks Gambling Screen). Los resultados obtenidos mostraron la presencia de un $4.18 \%$ de probables jugadores patológicos, un $6.39 \%$ de jugadores de riesgo, un $49.5 \%$ de jugadores sin problema y un $39,9 \%$ de sujetos que afirmaron no jugar. Respecto a las diferencias de género se han obtenido datos que indican la presencia de más varones que mujeres como jugadores patológicos, que los varones practican más clases de juegos que las mujeres, gastan más dinero en jugar en un solo día y son más criticados por jugar que ellas.
\end{abstract}

Palabras clave: Ludopatía; Jugador Patológico; Estudiantes Universitarios.

This study investigated the presence of pathological gambling characteristics in a sample of university students. In total, 908 students from different faculties at the University of Murcia (Spain) were assessed by answering the South Oaks Gambling Screen (SOGS). The results showed that $4.18 \%$ were probable pathological gamblers, $6.39 \%$ were at risk of pathological gambling, $49.5 \%$ could gamble without such risk, and $39.91 \%$ stated they never gambled. There were more male than female pathological gamblers and the male gamblers played more kinds of games than female gamblers. Male gamblers also spent more money on gambling per day than women and experienced more criticism for doing so than women.

Keywords: Gambling Behaviour; Pathological Gambling; University Students.

Correspondencia: Ma Angeles Jiménez Tallón. Departamento de Personalidad, Evaluación y Tratamiento Psicológicos. Facultad de Psicología. Universidad de Murcia. España. E-mail: ajtallon@um.es. E-Mail de los otros autores: Carmen García Montalvo: carmengm@um.es, Marian Montero Jiménez: marianmonteroj@hotmail.com, Ma del Carmen Perea Pérez: mcarmenpp4@hotmail.com. 
El juego en la infancia y adolescencia se considera como una actividad necesaria para el aprendizaje y desarrollo de las habilidades sociales de una persona, pero el juego también es una actividad lúdica a la que tienen acceso los adultos y que en ocasiones puede incorporar elementos que lo hacen más atractivo como el azar o la posibilidad de apostar.

Tradicionalmente se ha distinguido entre el juego como pasatiempo (play) y el juego con apuestas (gambling) que lleva implícita la posibilidad de arriesgar alguna ganancia. Si bien ambos tipos de juego pueden ser practicados de forma lúdica, es el juego con apuestas, de larga tradición histórica, el que ha facilitado la aparición de conductas problemáticas como el juego patológico o ludopatía.

En España se legalizó el juego en 1977. A partir de entonces fueron legales los bingos, casinos y máquinas tragaperras (consideradas como las mayores generadoras de adicción) (Secades y Villa, 1998).

El juego patológico se recoge por primera vez como categoría diagnóstica en el Manual Diagnóstico y Estadístico de los Trastornos Mentales, DSM-III, de la American Psychiatric Association (APA, 1980). Actualmente se utilizan para el diagnóstico del juego patológico los síntomas del DSM-IV-TR (2000) que son los mismos del DSM-IV (APA, 1994) para este trastorno. En la CIE-10 (Clasificación Internacional de Enfermedades de la Organización Mundial de la Salud, 1992), se define el juego patológico como el trastorno que consiste en la presencia de frecuentes y reiterados episodios de juegos de apuestas, que dominan la vida del enfermo en perjuicio de los valores y obligaciones sociales, laborales, materiales y familiares del mismo.

El curso de este trastorno se puede describir a partir de las fases propuestas por Custer (1984). El primer estadio sería la fase de ganancia, que se inicia con resultados positivos para el jugador que le invita a invertir más tiempo y dinero, favoreciendo pérdidas más numerosas que le llevan a una segunda etapa, la fase de pérdida. Ésta se caracteriza por usar el juego como estrategia para recuperarse de las pérdidas, pero para ello precisa dinero. Si las fuentes legales de préstamos se agotan, surge el riesgo de buscar el dinero de forma ilegal, las deudas aumentan y el jugador se ve obligado a confesar su situación implorando dinero con la promesa de dejar de jugar, pero una vez que dispone de dinero volverá a jugar y la mentira continuada a la familia y al terapeuta será una pauta de actuación. El cúmulo de pérdidas y deudas da lugar a la fase de desesperación, con un incremento de tiempo y dinero dedicados al juego. Se alcanza entonces la fase de desesperanza o abandono, propuesta por Lesieur y Rosenthal (1991).

En la actualidad existen numerosos estudios epidemiológicos sobre la prevalencia del juego patológico en diferentes países, así como en España, referidos a adultos, niños y adolescentes. Estos estudios se basan en los criterios diagnósticos del DSM-III, DSM-III-R o del SOGS, instrumento elaborado a partir de los criterios diagnósticos de ambos sistemas de clasificación.
En adultos, entre los estudios sobre la prevalencia del juego patológico, destacan los estudios pioneros de Custer (1982) y Culleton (1989) a los que siguieron estudios en Cánada (Ferris y Wynne, 2001; Ladoucer, 1991, 1996), Nueva Zelanda (Abbot y Volverg, 1996, 2000; Allcock, 1986), Australia (Dickerson, Baron, Hong y Cottrell, 1996; Dickerson, Cunningam, England y Hinchy, 1991), Reino Unido (Sproston, Erens y Orford, 2000), Suecia (Berg y Kühlhrn, 1994; Rönnberg, 2000), Hong Kong (Wong y So, 2003). Sin embargo, donde más estudios de prevalencia se han realizado ha sido en Estados Unidos. El National Research Council (1999) recoge 33 estudios epidemiológicos y más recientemente en Alberta Gaming Research Institute un total de 52. La mayoría de estos estudios han sido realizados con el South Oaks Gambling Screen (SOGS) de Lesieur y Blume $(1987,1993)$ como instrumento de evaluación del juego patológico. La prevalencia estimada de juego patológico en Estados Unidos oscilaba entre el 1 y el 2\% (Volberg, 1989, 1990, 1993, 1996, 2002). En este caso los criterios utilizados fueron los del DSM-III, DSM-III-R o el SOGS que combina en sus preguntas síntomas de uno y otro.

En España se han llevado a cabo una serie de investigaciones sobre la prevalencia del juego patológico basadas en el DSM-III, DSM-III-R o en el SOGS. Así, Becoña (1991; 1993 ; 1999), en Galicia, encontró una prevalencia de 1,73\% de jugadores patológicos y de $1.60 \%$ de jugadores problema. Entre los estudios más representativos que utilizaron el SOGS, se encuentran el estudio de Cayuela (1990) en Cataluña, en el que encontró un $2.50 \%$ de jugadores patológicos o problema en la población adulta catalana. Legarda, Babio y Abreu (1992) en Sevilla, indicaron que había $1.7 \%$ de probables jugadores patológicos y $5.18 \%$ de probables jugadores problema; Echeburúa, Báez, Fernández-Montalvo y Páez (1994) en el País vasco, sugirieron bajar el punto de corte del SOGS de 5 a 4 puntos, obteniendo así un 2\% de jugadores patológicos. Becoña y Fuentes (1995), utilizando también esta vez el SOGS, obtuvieron una prevalencia del $1.36 \%$ de jugadores patológicos y $2.04 \%$ de jugadores problema. Tejeiro (1998) encontró un $1.90 \%$ de jugadores patológicos y un $3.80 \%$ de jugadores problema en una muestra representativa de Algeciras. Por último, Ramírez et al. (1999), realizaron un estudio en Andalucía, con una muestra representativa, obteniendo $1.63 \%$ de jugadores patológicos y $1.43 \%$ de jugadores problema.

Becoña, Labrador, Echeburúa, Ochoa y Vallejo (1995), extrapolando los datos de otros países junto a los mencionados, estimaron la tasa de jugadores patológicos de más de 18 años existentes en España en 1.50\% de jugadores patológicos y $2.50 \%$ de jugadores problema.

El DSM-IV se publicó en 1994 con nuevos síntomas para el diagnóstico del juego patológico, que son una combinación de algunos síntomas del DSM-III y DSM-III-R, junto al síntoma de escape o utilización del juego como escape de situaciones cotidianas (Lesieur y Rosenthal, 1991). También se considera en esta edición que para diagnosticar el juego patológico hay que 
descartar un posible episodio maniaco, así como que de nueve síntomas del DSM-III-R se pasa a 10 en el DSM-IV, subiendo el punto de corte de cuatro a cinco síntomas. Estos cambios son relevantes para los estudios epidemiológicos porque el SOGS, instrumento predominante en la evaluación del juego patológico, ya no se adecua completamente al DSM-IV y por ello, en la última década, hay autores que han hablado del problema de los falsos positivos con el SOGS (Abbott y Volberg, 1996; Culleton, 1989; Dyckerson, 1993; Ferris y Wynne, 2001; Lesieur, 2002; Stinchfield, 2002; Volberg, 1999). Esto indica que en la población general el SOGS sobreestima la prevalencia del juego patológico. Debido a esto, en Estados Unidos un grupo de expertos elaboraron un nuevo instrumento: el NODS (NORC DSM-IV Screen for Gambling Problems) basado en los criterios del DSM-IV (Gernstein et al., 1999). Con este instrumento se realizó un amplio estudio, con el que se encontró un $.90 \%$ de jugadores patológicos a lo largo de la vida y un .30\% en el último año, resultados muy inferiores a los obtenidos con el SOGS. El porcentaje de jugadores problema también es muy inferior a los datos anteriores (Volberg, 1996, 2002).

Otros estudios realizados en los últimos años y en diferentes países, han utilizado nuevos instrumentos de evaluación del juego patológico o han revisado el SOGS, bajando el punto de corte. Abbott y Volberg (2000) obtuvieron con el SOGS-R un . $50 \%$ de jugadores patológicos en el último año; Rönnberg (2000) con el SOGS, un .60\%, Ferris y Wynne (2000), con el Canadian Problem Gambling Index (CPGI), encontraron un .70\%; Welte, Barnes, Wieczorek, Tidwell y Parker (2002) con el DSM-IV-DIS un $1.40 \%$; Wong y So (2003), con el Gambling Behavior Index (GBI), un 1.80\% y Becoña (2004) con el NODS en Galicia, obtiene un $.30 \%$ de jugadores patológicos en el último año. No obstante, varios autores han manifestado que el punto de corte de cinco síntomas del DSM-IV para diagnosticar el juego patológico es muy alto (Stinchfield, 2003) y también que el criterio de padecer el trastorno en los últimos doce meses para su diagnostico no significa que la persona no precise ayuda si tal criterio no se cumple (Toce-Gerstein y Gerstein, 2004).

En una relación de estudios realizados en los últimos años con diferentes metodologías y mostrados por Echeburúa, Becoña, Labrador y GAUDIUM (2010) se observa que la prevalencia del juego patológico en la población general es menor que la estimada hasta ahora, estando el rango entre el .10\% y el $.80 \%$ en casi todos ellos.

En jóvenes y adolescentes, el juego patológico presenta una prevalencia significativamente más elevada que en los adultos. Lesieur y Klein (1987) en el estado de Nueva York obtuvieron una prevalencia del juego patológico en estudiantes de enseñanza secundaria y universitaria entre un 4 y un $8 \%$ y en estudios más recientes se encontraron en EEUU entre un 3 $10 \%$ de jugadores problema.

Shaffer, LaBrie, Scaulan y Cummings (1994) hicieron un estudio referido al juego patológico en adolescentes y en un metaanálisis realizado posteriormente por Shaffer y Hall
(1996) sobre la prevalencia del juego patológico en adolescentes, incluyeron once estudios con sujetos de 13 a 20 años en los cuales, la prevalencia del juego compulsivo de alto riesgo fluctuó entre el $3.50 \%$ y el 17\%, la media estimada para el juego patológico o para graves problemas de juego oscilaba entre el 4.40 y el $7.40 \%$.

En España sólo se han realizado estudios parciales en adolescentes sobre el juego patológico, en los que suele hablarse de juego problema más que de juego patológico cuando se utilizan instrumentos derivados del SOGS, como el SOGS-RA en adolescentes. Sin embargo, estos datos no suelen correlacionar con el diagnóstico clínico siguiendo los criterios del DSM-IV-TR y sólo se hablaría de juego patológico al realizar un diagnóstico clínico con vistas a un tratamiento (Winters y Rich, 1998).

No son muy numerosos los estudios de juego patológico en estudiantes universitarios, (véase Griffiths, 1991; Ladoucer, Dube y Bujold, 1994; Lesieur y Klein, 1987). García y Correa (1998), aplicando el SOGS, obtuvieron un $2.70 \%$ de prevalencia de juego patológico y $5 \%$ de juego problema. Villoria, $(1999,2003)$ utilizando también el SOGS en una muestra de 1707 estudiantes de las universidades madrileñas, encontró un $4.50 \%$ de probables jugadores patológicos y un $6.60 \%$ de jugadores problema. Más recientemente en el estudio realizado por Cano-Bedoya y Pérez-Pérez (2008) en la Universidad de Medellín, con una muestra de 461 estudiantes y en el meta-análisis llevado a cabo por Muñoz-Molina (2008) sobre las investigaciones de prevalencia de la ludopatía realizadas entre 1997 y 2007, aparecen citados diversos estudios con muestras de adolescentes y estudiantes, realizados con el SOGS, cuyas cifras de prevalencia del juego patológico oscilan entre un 5.60\% y un $2.60 \%$.

Respecto al tipo de juegos practicados, la mitad de los jugadores patológicos comienzan jugando sólo a las máquinas tragaperras y de forma secundaria al bingo y a las cartas (Bombin, 1992). Los juegos de cartas con apuestas son uno de los juegos de azar más utilizados entre la población universitaria, al cumplir una función social en el contexto estudiantil, es el juego más practicado por los jugadores sociales cuyas pérdidas "controladas" van a depender de su poder adquisitivo. Por tanto, para la población estudiantil las cartas con apuestas adquieren un gran poder adictivo cuya gravedad dependerá del tipo de apuestas y de la ausencia de límites en las cantidades apostadas.

Este trabajo tiene por objetivo investigar la prevalencia de juego patológico en una muestra de estudiantes universitarios de la Universidad de Murcia, que cuenta con 27000 alumnos matriculados en primer y segundo ciclos e indagar sobre la problemática del jugador patológico universitario: conocer el perfil de este tipo de jugadores, las diferencias de género que pueda haber en las actividades de juego, así como la posible influencia familiar en la conducta de juego. Como punto de partida, asumimos que las actividades de juego patológico en este colectivo responden a la descripción de las características de ludopatía en 
sujetos que practican el juego, que los datos obtenidos de probables jugadores patológicos y jugadores problema o de riesgo serán más elevados que los de la población general, que los varones de esta muestra juegan más, gastan más dinero y tienen más problemas con el juego que las mujeres y, por último, que la familia influye en la adquisición de los hábitos de juego.

\section{Método}

\section{Participantes}

La muestra inicial estuvo formada por 976 participantes matriculados en diferentes facultades de la Universidad de Murcia. Fueron seleccionados a través de un muestreo incidental e intencional y los criterios de inclusión fueron: ser estudiante universitario y tener una edad entre 18 y 30 años. Los criterios de exclusión fueron: protocolos cumplimentados de forma incorrecta y los que aparecieron sin datos relativos al sexo o edad. En total fueron excluidos 68 participantes quedando definitivamente 908, 347 fueron varones y 561 mujeres.

\section{Procedimiento}

Un equipo de psicólogos y estudiantes de $5^{\circ}$ curso de Psicología, colaboradores en la investigación, tras explicar el objetivo de la investigación a cada grupo de estudiantes, aplicaron el South Oaks Gambling Screen en las diferentes facultades. El cuestionario fue cumplimentado a lo largo del curso 20072008 y 2008-2009.

En el cuestionario están incluidos datos relativos a la edad, el sexo, la carrera estudiada y un ítem sobre asistencia a asociaciones de rehabilitación de juego patológico. De la muestra estudiada se habían excluido previamente 68 cuestionarios por no resultar válidos, por tanto los cuestionarios aplicados inicialmente fueron 976 .

\section{Instrumentos}

A los 908 estudiantes se les aplicó el South Oaks Grambling Screen (SOGS) de Lesieur y Blume (1987), concretamente la adaptación española de Echeburúa, Báez, Fernández-Montalvo y Páez. (1994). Este cuestionario consta de 16 ítems.

El SOGS es el cuestionario más difundido y utilizado en la evaluación del juego patológico. Es un instrumento fiable ya que tiene una alta consistencia interna (coeficiente alfa de Cronbach $=.97 ; p=.001)$ y una alta correlación test-retest $(r$ $=.71 ; p<.01)$.

El SOGS no sólo permite detectar jugadores patológicos, sino que también identifica personas en situación de riesgo, por lo que facilita una intervención preventiva y terapéutica temprana. Así, el interés del SOGS radica en que permite una detección más precoz que los criterios diagnósticos del DSMIV-TR, que están referidos a una fase ya avanzada del juego patológico. Su limitación principal consiste en que se trata de una prueba de screening, útil para llevar a cabo una primera aproximación a poblaciones de riesgo, pero demasiado genérica como para posibilitar un análisis funcional detallado de los problemas concretos de cada sujeto. En esta prueba el punto de corte propuesto por los autores y que discrimina a los jugadores patológicos de los que no lo son, es de 5 o más respuestas afirmativas, que indican que el encuestado es un probable jugador patológico. En la presente investigación se optado por la utilización de este punto de corte, en lugar del de 4 que aplicaron los autores de la validación española inicialmente. Esta opción se ha basado principalmente en que ha sido el punto de corte utilizado en la mayoría de los estudios realizados con el SOGS y, también, por su contribución a contrarrestar la sobreestimación de la prevalencia de jugadores patológicos.

La validez del SOGS fue examinada correlacionando con evaluaciones independientes y diagnósticos basados en el DSMIII-R $(r=.94)$. La eficacia diagnóstica de este cuestionario, con el criterio original de 5 o más puntos, da una sensibilidad del 95\% y una especificidad del 98\% (Salinas, 2004).

Los ítems del SOGS utilizados son los siguientes: 1) juegos practicados, 2) cantidad de dinero gastado en un solo día, 3) familiares jugadores, 4) volver a jugar para recuperar el dinero perdido, 5) negar que había perdido, 6) tener problemas con el juego, 7) jugar más dinero del pensado, 8) ser criticado por jugar, 9) sentirse culpable por jugar, 10) le gustaría dejar de jugar, pero no lo hace, 11) ocultar que juega, 12) discutir con la familia por dinero, 13) discutirlo con la familia por causa del juego, 14) pedir dinero prestado para el juego y no devolverlo, 15) pérdida de tiempo para otras actividades por el juego, y 16) pedir dinero prestado para jugar o pagar deudas.

\section{Análisis estadístico}

\section{Resultados}

Los datos se analizaron mediante el programa estadístico SPSS-15. En el análisis de la muestra de 908 estudiantes aparecieron 38 probables jugadores patológicos (con 5 o más ítems afirmativos), de los cuales 30 eran varones y 8 mujeres; 58 jugadores problema o de riesgo (con 3 ó 4 ítems afirmativos), de los cuales 39 eran varones y 19 mujeres; 449 jugadores sin problema, de los cuales 180 eran varones y 269 mujeres y, finalmente, 363 no jugadores, de los que 98 eran varones y 265 mujeres.

En conclusión, en la muestra estudiada aparecieron un $4,18 \%$ de probables jugadores patológicos, un $6.39 \%$ de jugadores problema o de riesgo, un $49.45 \%$ de jugadores sin problema y un $39.99 \%$ que declararon no jugar.

Tabla 1

Porcentaje de Jugadores patológicos y de riesgo en la muestra estudiada $(N==908)$.

\begin{tabular}{lcccccc}
\hline \multicolumn{1}{c}{ Varones } & & \multicolumn{5}{c}{ Mujeres } \\
& $n$ & $\%$ & $n$ & $\%$ & Total & $\%$ \\
Juego patológico & 30 & 8.67 & 8 & 1.42 & 38 & 4.18 \\
Juego riesgo & 39 & 11.27 & 19 & 3.38 & 58 & 6.39 \\
Juego sin problemas & 180 & 52.02 & 269 & $47.95 \%$ & 449 & 49.45 \\
No juegan & 98 & 28.24 & 265 & $47.23 \%$ & 363 & 39.99 \\
Total & 347 & & 561 & & 908 & \\
\hline
\end{tabular}


Tabla 2

Análisis de componentes principales con rotación varimax $(N=908)$.

\begin{tabular}{|c|c|c|c|c|c|}
\hline Variables & Perfil jugador patológico & Problemas con el juego & Préstamos por juego & Asociaciones de juego & Antecedentes familiares \\
\hline Tipos de juego & .74 & & & & \\
\hline Disfrutar jugando & .82 & & & & \\
\hline Jugar más dinero & .81 & & & & \\
\hline Si pierde juega más & .58 & & & & \\
\hline Juega más de lo que pensó & .60 & & & & \\
\hline Perder tiempo jugando & .45 & & & & \\
\hline Oculta si pierde & & .62 & & & \\
\hline Problemas con el juego & & .52 & & & \\
\hline Criticado por jugar & & .60 & & & \\
\hline Se siente mal por jugar & & .51 & & .43 & \\
\hline No quiso pero jugó & & .55 & & & \\
\hline Oculta que juega & & .66 & & & \\
\hline Discutir por jugar & & & .41 & & \\
\hline Préstamos no devueltos & & & .79 & & \\
\hline Préstamos para jugar & & & .75 & & \\
\hline Ir a asociaciones de juego & & & & .86 & \\
\hline Familiares que juegan & & & & & .91 \\
\hline Género & & & & & .40 \\
\hline Varianza explicada & $19 \%$ & $15 \%$ & $11,56 \%$ & $7,14 \%$ & $6 \%$ \\
\hline Porcentaje total de varianza & plicada: $58.7 \%$ & & & & \\
\hline
\end{tabular}

Se realizó análisis factorial de componentes principales con rotación Varimax, con el objeto de seleccionar el número de factores interpretativos. Aparecieron 5 factores que explican el $58.7 \%$ de la varianza: Factor 1: Perfil del jugador patológico, Factor 2: Problemas con el juego, Factor 3: Préstamos para juego, Factor 4: Asociaciones de juego y Factor 5: Antecedentes familiares.

Los factores obtenidos en la factorización y los niveles de saturación se muestran en la tabla 2 .

Para conocer si hay diferencias entre varones y mujeres de la muestra, se aplicó un análisis discriminante paso a paso, tomando como variable dependiente el género, para conocer las variables que más discriminan a los varones y mujeres de la muestra y dio como resultado las siguientes variables por este orden: más dinero gastado en juego, más juegos practica- dos, ser criticado por jugar, tener familiares que juegan, ir a asociaciones de juego y ocultar que se juega. Según las matrices de clasificación, el $65.90 \%$ de los sujetos fue incluido en su grupo de referencia. En función de estos datos, los varones gastan más dinero en jugar en un solo día, practican más juegos, son más criticados por jugar, tienen más familiares que juegan y ocultan que juegan, en comparación con las mujeres.

Se realizó también un análisis de regresión lineal múltiple con el género como V.D. para conocer las variables predictoras de actividades de juego que diferencian varones de mujeres. Estas variables predictoras fueron: más dinero gastado en juego, más juegos practicados, ser criticado por jugar, tener familiares que juegan, ir menos a asociaciones de juego y ocultar que juegan.

Tabla 3

Análisis de regresión múltiple por pasos. VD Género. Variables predictoras de juego en función del género.

\begin{tabular}{|c|c|c|c|c|c|}
\hline Paso & Variables & Beta & $R^{2}$ & $F$ & $t$ \\
\hline 1 & Jugar más dinero & .31 & .09 & $99.19 *$ & $9.95 * *$ \\
\hline 2 & $\begin{array}{l}\text { Jugar más dinero } \\
\text { Jugar más juegos }\end{array}$ & $\begin{array}{l}.22 \\
.16\end{array}$ & .11 & $60.25 * *$ & $\begin{array}{l}6.19 * * \\
4.39 * *\end{array}$ \\
\hline 3 & $\begin{array}{l}\text { Jugar más dinero } \\
\text { Jugar más juegos } \\
\text { Criticado por jugar }\end{array}$ & $\begin{array}{l}.19 \\
.14 \\
.10\end{array}$ & .12 & $43.43 * *$ & $\begin{array}{l}5.23^{* *} \\
3.87^{* *} \\
2.96^{* *}\end{array}$ \\
\hline 4 & $\begin{array}{l}\text { Jugar más dinero } \\
\text { Jugar más juegos } \\
\text { Criticado por jugar } \\
\text { Familiares que juegan }\end{array}$ & $\begin{array}{l}.19 \\
.14 \\
.09 \\
.08\end{array}$ & .13 & $34.45 * *$ & $\begin{array}{l}5.07 * * \\
3.90^{* *} \\
2.76^{* *} \\
2.59^{* *}\end{array}$ \\
\hline 5 & $\begin{array}{l}\text { Jugar más dinero } \\
\text { Jugar más juegos } \\
\text { Criticado por jugar } \\
\text { Familiares que juegan } \\
\text { Ir a asociaciones de juego }\end{array}$ & $\begin{array}{l}.20 \\
.14 \\
.09 \\
.08 \\
-.06\end{array}$ & .13 & $28.54 * *$ & $\begin{array}{r}5.25^{* *} \\
3.90^{* *} \\
2.87 * * \\
2.74 * * \\
-2.09 * *\end{array}$ \\
\hline 6 & $\begin{array}{l}\text { Jugar más dinero } \\
\text { Jugar más juegos } \\
\text { Criticado por jugar } \\
\text { Familiares que juegan } \\
\text { Ir a asociaciones de juego } \\
\text { Ocultar juego }\end{array}$ & $\begin{array}{l}.19 \\
.14 \\
.08 \\
.08 \\
-.07 \\
.07\end{array}$ & .14 & $24.69 * *$ & $\begin{array}{l}5.21^{* *} \\
3.81^{* *} \\
2.43^{*} \\
2.63^{*} \\
-2.31 \\
2.19\end{array}$ \\
\hline
\end{tabular}

$* * p<.01 ; * p<.05$ 
Tabla 4

Comparación de medias mediante $t$ de Student entre Varones y Mujeres.

\begin{tabular}{|c|c|c|c|c|}
\hline Variables & Género & $M$ & $D T$ & $t$ \\
\hline Juegos practicados & $\begin{array}{l}\text { Mujer } \\
\text { Varón }\end{array}$ & $\begin{array}{l}1.65 \\
2.37\end{array}$ & $\begin{array}{c}.98 \\
1.42\end{array}$ & $\begin{array}{l}-8.89 * * \\
-8.10 * *\end{array}$ \\
\hline Disfruta más con el juego & $\begin{array}{l}\text { Mujer } \\
\text { Varón }\end{array}$ & $\begin{array}{r}.63 \\
1.02\end{array}$ & $\begin{array}{l}.81 \\
.94\end{array}$ & $\begin{array}{l}-6.50^{* *} \\
-6.24 * *\end{array}$ \\
\hline Gasta más dinero en jugar & $\begin{array}{l}\text { Mujer } \\
\text { Varón }\end{array}$ & $\begin{array}{r}.55 \\
1.09\end{array}$ & $\begin{array}{l}.66 \\
.86\end{array}$ & $\begin{array}{l}-10.37 * * \\
-9.70^{* *}\end{array}$ \\
\hline Más familiares que juegan & $\begin{array}{l}\text { Mujer } \\
\text { Varón }\end{array}$ & $\begin{array}{l}1.47 \\
1.60\end{array}$ & $\begin{array}{l}.49 \\
.48\end{array}$ & $\begin{array}{l}-3.62 * * \\
-3.64 * *\end{array}$ \\
\hline Si pierde vuelve a jugar & $\begin{array}{l}\text { Mujer } \\
\text { Varón }\end{array}$ & $\begin{array}{l}.20 \\
.45\end{array}$ & $\begin{array}{l}.48 \\
.70\end{array}$ & $\begin{array}{l}-6.30 * * \\
-5.75 * *\end{array}$ \\
\hline Oculta que perdió jugando & $\begin{array}{l}\text { Mujer } \\
\text { Varón }\end{array}$ & $\begin{array}{l}.03 \\
.16\end{array}$ & $\begin{array}{l}.20 \\
.41\end{array}$ & $\begin{array}{l}-6.15^{* *} \\
-5.23^{* *}\end{array}$ \\
\hline Problemas con el juego & $\begin{array}{l}\text { Mujer } \\
\text { Varón }\end{array}$ & $\begin{array}{l}.02 \\
.07\end{array}$ & $\begin{array}{l}.19 \\
.32\end{array}$ & $\begin{array}{l}-2.68^{*} \\
-2.36^{*}\end{array}$ \\
\hline Juega más de lo que pensó & $\begin{array}{l}\text { Mujer } \\
\text { Varón }\end{array}$ & $\begin{array}{l}1.08 \\
1.26\end{array}$ & $\begin{array}{l}.30 \\
.45\end{array}$ & $\begin{array}{l}-6.98^{* *} \\
-6.30^{* *}\end{array}$ \\
\hline Es criticado por jugar & $\begin{array}{l}\text { Mujer } \\
\text { Varón }\end{array}$ & $\begin{array}{l}1.02 \\
1.13\end{array}$ & $\begin{array}{l}.15 \\
.34\end{array}$ & $\begin{array}{l}-6.83^{* *} \\
-5.74 * *\end{array}$ \\
\hline Se siente mal por jugar & $\begin{array}{l}\text { Mujer } \\
\text { Varón }\end{array}$ & $\begin{array}{l}1.04 \\
1.11\end{array}$ & $\begin{array}{l}.22 \\
.32\end{array}$ & $\begin{array}{l}-3.84 * * \\
-3.49^{* *}\end{array}$ \\
\hline No quiso jugar pero lo hizo & $\begin{array}{l}\text { Mujer } \\
\text { Varón }\end{array}$ & $\begin{array}{l}1.05 \\
1.15\end{array}$ & $\begin{array}{l}.22 \\
.36\end{array}$ & $\begin{array}{l}-5.03^{* *} \\
-4.48^{* *}\end{array}$ \\
\hline Oculta que juega & $\begin{array}{l}\text { Mujer } \\
\text { Varón }\end{array}$ & $\begin{array}{l}1.02 \\
1.10\end{array}$ & $\begin{array}{l}.14 \\
.30\end{array}$ & $\begin{array}{l}-5.41^{* *} \\
-4.58^{* *}\end{array}$ \\
\hline Discute por el juego & $\begin{array}{l}\text { Mujer } \\
\text { Varón }\end{array}$ & $\begin{array}{l}1.01 \\
1.04\end{array}$ & $\begin{array}{l}.12 \\
.21\end{array}$ & $\begin{array}{l}-2.85^{*} \\
-2.50^{*}\end{array}$ \\
\hline Pierde tiempo jugando & $\begin{array}{l}\text { Mujer } \\
\text { Varón }\end{array}$ & $\begin{array}{l}1.05 \\
1.17\end{array}$ & $\begin{array}{l}.22 \\
.38\end{array}$ & $\begin{array}{l}-6.17 * * \\
-5.42 * *\end{array}$ \\
\hline Pide préstamos para jugar & $\begin{array}{l}\text { Mujer } \\
\text { Varón }\end{array}$ & $\begin{array}{l}1.08 \\
1.13\end{array}$ & $\begin{array}{l}.27 \\
.34\end{array}$ & $\begin{array}{l}-2.58^{*} \\
-2.43^{*}\end{array}$ \\
\hline
\end{tabular}

$* * p<.01 * p<.05$

Para estimar si existían diferencias estadísticamente significativas en actividades de juego entre los varones y mujeres de la muestra, se aplicó la $t$ de Student para la variable género, esto permitió conocer las siguientes diferencias significativas, para un nivel de significación del 1\% y del 5\%:

Los varones y mujeres de la muestra se diferencian significativamente en que los varones practican más juegos, disfrutan más jugando, gastan más cantidad de dinero en el juego, tienen más familiares que juegan, si pierden vuelven a jugar, ocultan que perdieron jugando, tienen más problemas con el juego, juegan más de lo pensado, son más criticados por jugar, se sienten mal por jugar, no quisieron jugar pero lo hicieron, ocultan más que juegan, discuten más con la familia por el juego y pierden más tiempo de otras actividades por el juego.

No se apreciaron diferencias significativas entre varones y mujeres en pedir préstamos para jugar y no devolverlos.

Para conocer las diferencias entre los sujetos con familiares jugadores y sin familiares jugadores se aplicó un segundo análisis discriminante paso a paso, con variable dependiente: familiares que juegan, que dio como resultado que las variables que más discriminaban fueron, por este orden: si pierde vuelve a jugar, predominan los varones, disfrutan menos jugando que los que no tienen familiares jugadores, no quieren jugar pero lo hacen y van a Asociaciones de juego más que los que no tienen familiares jugadores. Según las matrices de clasificación, fueron clasificados correctamente el $57.10 \%$ de los casos.

Según los datos citados, los sujetos con familiares que juegan, si pierden vuelven a jugar, los varones son más influenciables al modelo familiar de juego, disfrutan menos jugando, no quieren jugar pero lo hacen y van a Asociaciones de juego más que los que no tienen familiares que juegan.

También se aplicó un análisis de regresión lineal múltiple con familiares jugadores como V.D., para conocer las variables predictoras de juego de los sujetos con familiares que juegan y se obtuvieron las siguientes variables predictoras de juego por este orden: Si pierde vuelve a jugar, ser varón, no disfruta jugando, no quería jugar pero lo hizo e ir a asociaciones de juego.

Según los datos citados, los sujetos con familiares jugadores, si pierden vuelven a jugar, los varones son más influenciables al modelo familiar de juego, disfrutan menos jugando que los que no tienen familiares jugadores, no quieren jugar pero lo hacen y van más a Asociaciones de juego que los que no tienen familiares que juegan.

Tabla 5. Análisis de Regresión múltiple por pasos. VD familiares que juegan. Variables predictoras de juego en sujetos con familiares jugadores.

\begin{tabular}{clcccc}
\hline Paso & \multicolumn{1}{c}{ Variables } & Beta & $R^{2}$ & $F$ & $t$ \\
1 & Si pierde juega & .13 & .19 & $17.08^{* *}$ & $4.13^{* *}$ \\
2 & Si pierde juega & .11 & .02 & $12.95^{* *}$ & $3.51^{* *}$ \\
& Género & .09 & & & $2.94^{* *}$ \\
3 & Si pierde juega & .17 & .03 & $11.17^{* *}$ & $4.44^{* *}$ \\
& Género & .10 & & & $3.24 * *$ \\
& Disfruta jugando & -.10 & & & $-2.72^{* *}$ \\
4 & Si pierde juega & .14 & .04 & $9.99^{* *}$ & $3.42^{* *}$ \\
& Género & .10 & & & $3.02^{* *}$ \\
& Disfruta jugando & -.11 & & & $2.93^{* *}$ \\
& No quiso jugar pero lo hizo & .09 & & & $2.50^{*}$ \\
5 & Si pierde juega & .13 & .04 & $8.98^{*}$ & $3.31^{* *}$ \\
& Género & .10 & & & $3.11^{* *}$ \\
& Disfruta jugando & -.11 & & & $-2.91^{* *}$ \\
& No quiso jugar pero lo hizo & .08 & & & $2.20^{*}$ \\
& Ir a asociaciones de juego & .07 & & & $2.18^{*}$ \\
\hline
\end{tabular}

$* p<.05 ; * * p<.01$ 


\section{Discusión}

Los resultados de este trabajo han permitido confirmar que tanto el perfil del probable jugador patológico de esta muestra como su problemática personal y psicológica se ajustan a la descripción de las características de ludopatía en sujetos que practican el juego.

Se puede apreciar que la prevalencia en esta investigación es superior a la informada en otros estudios sobre la población general española: un $4.18 \%$ de probables jugadores patológicos y $6.39 \%$ de jugadores problema o de riesgo; no obstante, se ha de tener en cuenta que los porcentajes de prevalencia suelen ser más elevados en la población joven y en la universitaria. También ha de tenerse en cuenta que el instrumento de evaluación ha sido el SOGS que, como se ha dicho, parece que sobreestima las características de ludopatía. Sin embargo, los datos obtenidos por Villoria (2003) con estudiantes de la Universidad Complutense de Madrid (4.50\% probables jugadores patológi$\cos$ y $6.60 \%$ jugadores problema), se aproximan bastante a los obtenidos en la Comunidad de Murcia.

Respecto a las diferencias de género, se han obtenido diferencias significativas que confirman la hipótesis inicial de que los varones de la muestra son más jugadores que las mujeres: practican más juegos, disfrutan más jugando, gastan más cantidad de dinero en el juego, tienen más familiares que juegan, si pierden vuelven a jugar, ocultan más cuando pierden jugando, tienen más problemas con el juego, juegan más de lo pensado, son más criticados por jugar, se sienten mal por jugar, no quieren jugar en ocasiones pero lo hacen, ocultan más que ellas que juegan, discuten más con la familia por el juego y pierden más tiempo de otras actividades por el juego, que ellas, sin embargo no se han obtenido diferencias significativas entre varones y mujeres en pedir préstamos para jugar y no devolverlos.

En cuanto a jugadores con familiares que juegan y su influencia en las actitudes de juego, cerca del $40 \%$ de los jugadores patológicos tienen antecedentes de juego patológico o de otro tipo de adicciones en su familia, que en ocasiones se extienden hasta la tercera generación (Garrido, Jaén y Domínguez, 2004). La familia, por tanto, parece que desempeña un papel muy importante en la adquisición de hábitos de juego y un $86 \%$ de los adolescentes que juegan, lo hacen con sus familiares (Gupta y Dervensky, 1997). En este trabajo también se ha confirmado la relación entre familiares que juegan y hábitos de juego.

Otros estudios han destacado que el déficit de comunicación y la pobreza relacional de las familias, serían variables que impulsarían a los adolescentes a jugar, aunque no hubiera un antecedente explícito de juego en sus familiares de origen (Yeoman y Griffiths, 1996). Asimismo, el deterioro de las relaciones de pareja y de otras relaciones familiares serían un elemento relevante para comprender las conductas del juego patológico (Garrido et al., 2004). La relación entre el jugador patológico y las pautas de relación familiar no entran en una lógica etiológica sino que es en el marco de la propia familia donde los problemas de comunicación y los estilos disfunciona- les de relación contribuyen al desarrollo de la conducta de juego.

Un estudio realizado en Murcia en 2004 con una muestra de 256 adolescentes, concluyó que las características familiares estaban relacionadas con los riesgos de ludopatía que presentaban una parte de los sujetos estudiados (Jiménez-Tallón, García-Montalvo, López-Soler y Dólera, 2004). Lo que está claro, es que el impacto del juego patológico va más allá de la persona afectada y son numerosas las consecuencias observadas en la familia que han sido descritas por diferentes autores (Fernández-Montalvo y Castillo, 2004), no obstante, hasta el momento no existen estudios suficientemente rigurosos sobre esta temática.

Tras el análisis de resultados podemos concluir, que el perfil del probable jugador patológico de esta muestra es: con más frecuencia es un varón que practica diferentes juegos, disfruta jugando, gasta bastante dinero en un día, si pierde vuelve a jugar, juega más de lo pensado y pierde tiempo de otras actividades por el juego.

En relación a la problemática del jugador de la muestra: tiene problemas con el juego, oculta cuando pierde, es criticado por jugar, se siente mal por hacerlo, no quiere jugar pero lo hace y discute con la familia por el juego.

Respecto a las diferencias de género encontradas se puede decir que los varones practican más clases de juegos que las mujeres, disfrutan más jugando, gastan más dinero en jugar en un solo día, tienen más familiares que juegan, si pierden vuelven a jugar, ocultan con mayor frecuencia que pierden jugando, tienen más problemas con el juego, juegan más de lo pensado, les critican más por jugar que a las mujeres, se sienten mal por jugar, no quieren jugar pero lo hacen, ocultan más que juegan, discuten más con la familia y pierden más tiempo de otras actividades por el juego que ellas.

En relación a la influencia familiar en las actitudes de juego en este trabajo hemos podido apreciar que la familia influye en los hábitos de juego, pues aquellos que tienen familiares que juegan, gastan más dinero en el juego, son más influenciables respecto al modelo familiar de juego, mayoritariamente los varones, si pierden vuelven a jugar, aunque no quieran jugar acaban haciéndolo, disfrutan menos jugando y han ido a asociaciones de juego más que los que no tienen familiares que juegan.

Según las conclusiones de este estudio, la población universitaria, tan amplia en nuestro país, puede ser población de riesgo de este importante problema social. Por este motivo, es necesario seguir investigando para conocer mejor los factores ambientales y situacionales que rodean los juegos de azar y así poder determinar con mayor rigor qué variables son las que intervienen en los procesos de adquisición de este tipo de adicciones. De esta forma se podrán elaborar programas de prevención en estos grupos de mayor riesgo. Además, el estudio de las repercusiones familiares, poco estudiado todavía, es muy necesario para poder ofrecer un tratamiento integral de la ludopatía. 


\section{Referencias}

1. Abbot, M. V. y Volberg, R. A. (1996). The New Zealand National Survey of Problem and Pathological Gambling. Journal of Gambling Studies, 12, 143-160. http://dx.doi. org/10.1007/BF01539171

2. Abbot, M. V. y Volberg, R. A. (2000). Prevalence of pathological gambling in New Zealand. Paper presented at the $11^{\text {th }}$. International Conference on Gambling \& Risk Taking. Las Vegas, Nevada.

3. Allcock C. C. (1986). Pathological Gambling. Australian and New Zealand Journal of Psychiatry, 20, 259-265. http://dx.doi.org/10.3109/00048678609158874

4. American Psychiatric Association (1980). Diagnostic and statistical manual of mental disorders (3. ${ }^{\text {a }}$ edición) (DSMIII). Washington, DC: Autor.

5. American Psychiatric Association (1994). Diagnostic and statistical manual of mental disorders (4. ${ }^{\text {a edición) (DSM- }}$ IV). Washington, DC: Autor.

6. American Psychiatric Association (2000). Diagnostic and statistical manual of mental disorders $\left(4 .^{\text {a }}\right.$ edición-Texto revisado) (DSM-IV-TR). Washington, DC: Autor.

7. Becoña, E. (1991). The prevalence of pathological gambling in Galicia. Paper presented at the Society for the Study of Gambling. London

8. Becoña, E. (1993). El juego compulsivo en la Comunidad Autónoma Gallega. Santiago de Compostela: Xunta de Galicia.

9. Becoña, E. (1999). Epidemiología del juego patológico en España. Anuario de Psicología, 30, 7-19.

10. Becoña, E. (2004). Prevalencia del juego patológico en Galicia mediante el NODS. ¿Descenso de la prevalencia o mejor evaluación del trastorno?. Adicciones 16, 173-184.

11. Becoña, E. y Fuentes, M. J. (1995). El juego patológico en Galicia, evaluado con el South Oaks Gambling Screen. Adicciones, 7, 423-440.

12. Becoña, E., Labrador, F., Echeburúa, E., Ochoa, E. y Vallejo, M. A. (1995). Slot machine gambling in Spain: An important and new social problem. Journal of Gambling Studies, 11, 265-286. http://dx.doi.org/10.1007/BF02104793

13. Berg, C. y Kühlhorn, E. (1994). Social, psychological and physical consequences of pathological gambling in Sweden. Journal of Gambling Studies, 10, 275-285. http:// dx.doi.org/10.1007/BF02104968

14. Bombin, B. (1992). El juego de azar. Patología y testimonio. Valladolid: Junta de Castilla y León.

15. Cano-Bedoya, V. H. y Pérez-Pérez, J. A. (2008). La ludopatía. Una mirada desde la Psicología. El Agora USB, 8, 437-458.

16. Cayuela, R. (1990). Characteristics and situation of gambling addiction in Spain: Epidemiology and clinical aspects. Paper presented at the Eight International Conference on Risk and Gambling. London.

17. Culleton, R. P. (1989). The prevalence rates of pathological gambling: A look at methods. Journal of Gambling Behavior, 5, 22-41. http://dx.doi.org/10.1007/BF01022135

18. Custer, R. L. (1982). An overview of compulsive gambling. En P. J. Carone, S. F. Yoles, S. N. Kieffer y L. Krinsky (Eds.), Addictive disorders update: alcoholism drug abuse, gambling (pp. 107-124). New York: Human Science Press.

19. Custer, R. L. (1984). Profile of the pathological gambler. Journal of Clinical Psychiatry, 45, 2-12.

20. Dyckerson, M. (1993). Aproximaciones alternativas a la medición de la prevalencia del juego patológico. Psicología Conductual, 1, 339-349.

21. Dyckerson, M., Cunningam, R., England, S. L. y Hinchy, J. (1991). On the determinants of persistent gambling: III. Personality, prior mood, and poker machine play. International Journal of the Addictions, 26, 531-548.

22. Dyckerson, M. G., Baron, E., Hong, S-M. y Cottrell, D. (1996). Estimating the extent and degree of gambling related problems in the Australian population: A National Survey. Journal of Gambling Studies, 12, 161-178. http:// dx.doi.org/10.1007/BF01539172

23. Echeburúa, E., Báez, C., Fernández-Montalvo, J. y Páez, D. (1994). Cuestionario de juego patológico: South Screen Oaks Gambling (SOGS). Análisis y Modificación de Conducta, 20, 769-791.

24. Echeburúa, E., Becoña, E., Labrador, F. y GAUDIUM (Coords.) (2010). El juego patológico. Avances en la clínica y en el tratamiento. Madrid: Pirámide.

25. Fernández-Montalvo, J. y Castillo, A. (2004). Repercusiones familiares del juego patológico: Una revisión crítica. Salud y Drogas, 4, 149-166.

26. Ferris, J. y Winne, H. (2001). The Canadian problem gambling index: Final report. Recuperado de http://www.ccsa. ca/2003\%20and $\% 20$ earlier $\% 20$ CCSA $\% 20$ Documents/ ccsa-008805-2001.pdf.

27. García, E. y Correa, C. (1998). El juego patológico en estudiantes universitarios. Acta Psiquiátrica y Psicológica de América Latina, 44, 63-68

28. Garrido, M., Jaén, P. y Domínguez, A. (2004). Ludopatía y relaciones familiares. Barcelona: Paidós.

29. Gerstein, D., Murphy, S., Toce, M., Hoffman, J., Palmer, A., Johnson, R., Larison C, Chuchro L, Bard A, Engelman L, Hill MA, Buie T, Volberg R, Harwood H, Tucker A, Christiansen E, Cummings W, Sinclair S. (1999). Gambling impact and behavior study. Chicago: Report to the National Gambliers Impact Study Commission.

30. Griffiths, M. D. (1991). An analysis of "Amusement machines dependency and delinquence" (Home office Research Study 101). Journal of Gambling Studies, 7, 79-86. http:// dx.doi.org/10.1007/BF01019768

31. Gupta, R. y Dervensky, J. (1997). Familial and social influences on juvenile gambling behavior. Journal of Gambling Studies, 13, 179-192. http://dx.doi. org/10.1023/A:1024915231379 
32. Jiménez-Tallón, M. A., García-Montalvo, C., López-Soler, C. y Dólera, M. J. (2004, Abril). Evaluación psicosocial de las características de ludopatía en una muestra de adolescentes murcianos. Comunicación presentada en la VII European Psychological Assessment Conference y VI Congreso Nacional de Evaluación Psicológica. Málaga (España).

33. Ladoucer, R. (1991). Prevalence estimates of pathological gambling in Québec. Canadian Journal of Psychiatry, 36, 732-734.

34. Ladoucer, R. (1996). The prevalence of pathological gambling in Canada. Journal of Gambling Studies, 12, 129-142. http://dx.doi.org/10.1007/BF01539170

35. Ladoucer, R., Dube, D. y Bujold, A. (1994). Prevalence of pathological gambling and related problems among college students in the Quebec metropolitan area. Canadian Journal of Psychiatry, 39, 289-293.

36. Legarda, J. J., Babio, R. y Abreu, J. M. (1992). Prevalence estimates of pathological gambling in Sevilla (Spain). British Journal of Addictions, 87, 767-770. http://dx.doi. org/10.1111/j.1360-0443.1992.tb02722.x

37. Lesieur, H.R. (2002). Epidemiological surveys of pathological gamblers: Critique and suggestions for modification. En J. J. Morotta., J. A. Cornelius y W. R. Eadington (Eds.), The downside: Problem and pathological gambling (pp. 325-338). Reno, Nevada: Institute for the Study of Gambling \& Commercial Gamming.

38. Lesieur, H. R. y Blume, S. B. (1987). The South Oaks Gambling Screen (SOGS): A new instrument for the identification of pathological gamblers. American Journal of Psychiatry, 144, 1184-1188.

39. Lesieur, H. R. y Blume, S. B. (1993). Revising the South Oaks Gambling Screen in different settings. Journal of Gambling Studies, 9, 213-223. http://dx.doi.org/10.1007/ $\underline{B F 01015919}$

40. Lesieur, H. R. y Klein, R. (1987). Pathological gambling among high school students. Addictive Behaviors, 12, 129135. http://dx.doi.org/10.1016/0306-4603(87)90019-0

41. Lesieur, H. R. y Rosenthal R. J. (1991). Pathological gambling: A review of the literature prepared for the American Psychiatryc Association (Task force on DSM-IV. Committee on disorders of impulsive control not elsewhere classified). Journal of Gambling Studies, 7, 5-39. http:// dx.doi.org/10.1007/BF01019763

42. Muñoz-Molina, Y. (2008). Meta-análisis sobre juego patológico 1997-2007. Revista Salud Pública, 10, 150-159.

43. National Research Council (1999). Pathological Gambling: A critical review. Washington, DC: Autor.

44. Organización Mundial de la Salud (1992). CIE-10. Trastornos mentales y del comportamiento. Descripciones clínicas y pautas para el diagnóstico. Madrid: Meditor.

45. Ramírez, J. N., Cabanes, M., Carbonero, M, Castilla, R., Duarte, J., Gutierrez, B. et al. (1999). Análisis del sector de las máquinas recreativas "tipos $A$ y B". Estimación y análisis de la proporción de ludópatas en Andalucía. Córdoba: ANMARE.

46. Rönnberg, S. D. (2000). Pathological Gambling in Sweden. Paper presented at the 11th. International Conference on Gambling \& Risk Taking. Las Vegas, Nevada.

47. Salinas, J. M. (2004). Instrumentos de diagnóstico y screening del juego patológico. Salud y Drogas, 4, 35-39.

48. Secades, R. y Villa, A. (1998). El juego patológico. Prevención, evaluación y tratamiento en la adolescencia. Madrid: Pirámide.

49. Shaffer, H. J. y Hall, M. N. (1996). Estimating the prevalence of adolescent gambling disorders: a quantitative synthesis and guide toward standard gambling nomenclature. Journal of Gambling Studies, 12, 193-214. http:// dx.doi.org/10.1007/BF01539174

50. Shaffer, H. J., LaBrie, R., Scaulan, K. M. y Cummings, T. N. (1994). Pathological Gambling among adolescents: Massachusetts Gambling Screen (MAGS). Journal of Gambling Studies, 10, 339-362. http://dx.doi.org/10.1007/ BF02104901

51. Sproston, K., Erens, B. y Oxford, J. (2000). Gambling behaviour in Britain: Results from the British Gambling Prevalence Survey. Londres, RV: National Centre for Social Research.

52. Stinchfield, R. (2002). Reliability, validity and classification accuracy of the South Oaks Gambling Screen SOGS. Addictive Behaviors, 27, 1-19. http://dx.doi.org/10.1016/ S0306-4603(00)00158-1

53. Stinchfield, R. (2003). Reliability, Validity and classification accuracy of a measure of DSM-IV diagnostic criteria for Pathological Gambling. American Journal of Psychiatry, 160, 180-182. http://dx.doi.org/10.1176/appi. ajp.160.1.180

54. Tejeiro, R. (1998). Estudio sobre el juego de azar en Algeciras. Prevalencia y características sociodemográficas. Algeciras: JARKA.

55. Toce-Gerstein, M. y Gerstein, D. R. (2004). Of time and the chase: Lifetime versus pat-year measures of pathological gambling. e gambling. The Electronic Journal of Gambling Issues, 10, 1-5.

56. Villoria, C. (1999). Percepción social de los juegos de azar entre los universitarios: Factores ambientales en el juego patológico. Recuperado de http://psiquiatría.com/psicología/articulos/adicciones/921/

57. Villoria, C. (2003). El juego patológico en los universitarios de la Comunidad de Madrid. Clínica y Salud, 14, 43-65.

58. Volberg, R. A. (1989, junio). Prevalence rates of problem gambling in the United States. Paper presented at the Fourth National Conference on Compulsive Gambling.

59. Volberg, R. A. (1990, agosto). Estimating the Prevalence of Pathological Gambling in the United State. Paper presented at the Eight International Conference on Risk and 
Gambling. London.

60. Volberg, R. A. (1993). Estimating the prevalence of pathological gambling in the United States. En : V. R. Eadington y J. A. Cornelius (Eds.), Gambling behaviour and problem gambling (pp. 365-378). Reno, NE: Institute for the Studies of Gambling and Commercial Gaming.

61. Volberg, R. A. (1996). Prevalence Studies of problem gambling in the United States. Journal of Gambling Studies, 12, 111-128. http://dx.doi.org/10.1007/BF01539169

62. Volberg, R. A. (1999). Research Methods in the epidemiology of pathological gambling: Development of the field and directions for the future. Anuario de Psicología, 30, 33-46.

63. Volberg, R. A. (2002). The epidemiology of pathological gambling. Psychiatric Annals, 32, 171-178.

64. Welte, J. V., Barnes, G. M., Wieczorek, W. F., Tidwell, M.C., y Parker, J. (2002). Gambling participation in the U.S. Results from a National Survey. Journal of Gambling Studies, 18, 313-337. http://dx.doi.org/10.1023/A:1021019915591
65. Winters, K. y Rich, T. (1998). A twin study of adult gambling behaviour. Journal of Gambling Studies, 14, 213-225. http://dx.doi.org/10.1023/A:1022084924589

66. Wong, I. L. y So, E. M. (2003). Prevalence estimates of problem and pathological gambling in Hong Kong. American Journal of Psychiatry, 160, 1353-1354. http://dx.doi. org/10.1176/appi.ajp.160.7.1353

67. Yeoman, T. y Griffiths, M. (1996). Adolescent machine gambling and crime. Journal of Adolescente, 19, 98-104.

Fecha de recepción: 8 de marzo de 2011 Fecha de recepción de la versión modificada: 23 de mayo de 2011

Fecha de aceptación: 23 de julio de 2011 